\title{
Selection Criteria for Patients With Chronic Ankle Instability in Controlled Research: A Position Statement of the International Ankle Consortium
}

\author{
Phillip A. Gribble, PhD, ATC, FNATA*; Eamonn Delahunt, PhD, BSc†; \\ Christopher M. Bleakley, PhDł; Brian Caulfield, PhD, MSc†; Carrie L. \\ Docherty, PhD§; Daniel Tik-Pui Fong, PhDll; François Fourchet, PhD, PTף; \\ Jay Hertel, PhD, ATC, FACSM, FNATA\#; Claire E. Hiller, PhD**; Thomas W. \\ Kaminski, PhD, ATC, FACSM††; Patrick O. McKeon, PhD, ATC ¥¥; Kathryn M. \\ Refshauge, PhD**; Philip van der Wees, PhD, PT§§; William Vicenzino, PhD, \\ MSc, BPhty, Grad Dip Sports Phtyllll; Erik A. Wikstrom, PhD, ATC, FACSMTT
}

*The University of Toledo, OH; †University College Dublin, Ireland; †The University of Ulster, UK; §Indiana University, Bloomington; IILoughborough University, Leicestershire, UK; ПAspire Health Centre, National Sports Medicine Programme (NSMP), Doha, Qatar; \#The University of Virginia, Charlottesville; ${ }^{*}$ The University of Sydney, Australia; ††The University of Delaware, Newark; 抽e University of Kentucky, Lexington; §§Radboud University Nijmegen Medical Center, Netherlands; IIITThe University of Queensland, Brisbane, Australia; ПๆThe University of North Carolina at Charlotte

\begin{abstract}
While research on chronic ankle instability (CAI) and awareness of its impact on society and health care systems has grown substantially in the last 2 decades, the inconsistency in participant or patient selection criteria across studies presents a potential obstacle to addressing the problem properly. This major gap within the literature limits the ability to generalize this evidence to the target patient population. Therefore, there is a need to provide standards for patient or participant selection
\end{abstract}

criteria in research focused on CAI with justifications using the best available evidence. The International Ankle Consortium provides this position paper to present and discuss an endorsed set of selection criteria for patients with CAI based on the best available evidence to be used in future research and study designs. These recommendations will enhance the validity of research conducted in this clinical population with the end goal of bringing the research evidence to the clinician and patient.

\section{EPIDEMIOLOGY AND IMPACT OF ANKLE INJURY}

I njuries to the ankle joint account for $20 \%$ of the population that is afflicted with joint injury. ${ }^{1}$ There are more than 3 million emergency room visits annually for ankle and foot injuries in the United States, ${ }^{2}$ and the largest percentage of self-reported musculoskeletal injuries $(>10 \%)$ is to the ankle. ${ }^{3}$ More than 628000 ankle injuries, including ankle sprains and fractures, per year are treated in United States emergency rooms, accounting for $20 \%$ of all injuries treated in emergency facilities. ${ }^{4}$ Ankle sprains account for an estimated 3\% to $5 \%$ of emergency room visits in the United Kingdom, ${ }^{5}$ representing a significant amount of devoted health care resources. Additionally, it is estimated that as many as $55 \%$ of patients who sustain an ankle sprain do not seek evaluation or treatment from a health care professional. ${ }^{6}$ Subsequently, the reporting of traumatic ankle sprains may be grossly underreported in health care statistics.

\section{SHORT-TERM AND LONG-TERM SEQUELAE}

Traumatic ankle injury represents a significant health care issue. Of further significance is that ankle sprains have a high rate of recurrence (as high as $80 \%$ in high-risk sports). ${ }^{7-9}$ Recent data indicate that ankle sprains are not just an innocuous injury primarily incurred by young athletes but also impact approximately $8 \%$ of the general population who report persistent symptoms following an initial ankle sprain. ${ }^{10}$ Chronic joint injury and degeneration is associated with over $\$ 3$ billion in annual health care costs in the United States. ${ }^{11}$ Evidence for the relationship between acute and recurrent ankle joint trauma and the development of posttraumatic ankle joint osteoarthritis (OA) is growing. ${ }^{11,12}$ Saltzman et a ${ }^{13}$ have reported that as many as 4 in 5 cases of ankle joint OA are the result of previous musculoskeletal trauma, with these patients being on average a decade younger than patients with primary ankle joint OA. Additionally, self-reported disability using the SF-36 Physical Component Score was significantly 
lower in patients with ankle OA from the United States ${ }^{13}$ compared with the general population and was also equal to or lower compared with patients suffering end-stage kidney disease, ${ }^{14}$ chronic heart failure, ${ }^{15}$ or Parkinson disease. ${ }^{16}$ Therefore, ankle joint sprains and their associated sequelae affect patients across the lifespan and may represent a large health care burden.

\section{ADVANCES IN RESEARCH}

The prevalence and impact of ankle sprains on society and health care systems support the need for continued research related to the prevention, treatment, and rehabilitation of ankle sprains and their associated sequelae. As mentioned above, an unfortunate and prominent consequence of acute ankle sprains is a very high recurrence rate. It has been reported that $32 \%$ to $74 \%$ of individuals with a previous history of ankle sprain suffer from some type of residual and chronic symptoms, recurrent ankle sprains, and/or perceived instability. ${ }^{17,18}$ Evidence from peerreviewed literature suggests that the characteristics of patients with recurrent ankle injury are not homogeneous. Many categorical descriptions have been used to define this pathology, including chronic ankle instability (CAI), functional ankle instability, mechanical ankle instability, and recurrent ankle instability. ${ }^{19-21}$ Chronic ankle instability has been defined in a variety of ways but is most predominantly described "as an encompassing term used to classify a subject with both mechanical and functional instability of the ankle joint." 20

\section{INTERNATIONAL ANKLE CONSORTIUM POSITION STATEMENT}

The International Ankle Consortium is an international community of researchers and clinicians whose primary scholastic purpose is to promote scholarship and dissemination of research-informed knowledge information related to pathologies of the ankle complex. The constituents of the International Ankle Consortium and other similar organizations have yet to properly define the clinical phenomenon known as CAI and its related characteristics for consistent patient recruitment and advancement of research in this area. While research on CAI and awareness of its impact on society and health care systems has grown substantially in the last 2 decades, the inconsistency in participant or patient selection criteria across studies presents a potential obstacle to addressing the problem properly. This major gap within the literature limits the ability to generalize this evidence to the target patient population. Therefore, there is a need to provide standards for patient or participant selection criteria in research focused on CAI with justifications using the best available evidence. The primary rationale for documenting such standards is to outline specific inclusion criteria that should be reported upon as a minimum when conducting research in the area of CAI. This will be of particular importance as research into CAI continues to grow and become more sophisticated, especially to enable high-fidelity synthesis and meta-analyses of data through future systematic reviews.

Although CAI is a multifaceted condition, there have been research developments to capture functional deficits associated with those who have recurrent issues. Freeman et $\mathrm{al}^{22}$ were among the first to recognize measurable differences in clinical outcomes in patients that had a history of ankle joint injury. Recognition of prolonged deficits in single-limb balance after ankle ligament sprains led to a theory of changes in neural signaling following trauma to the ankle joint and the categorization of these patients as having functional ankle instability. Several decades later, Herte ${ }^{19}$ presented a model that recognized the contributions from functional and mechanical insufficiencies associated with an acute ankle sprain that may interact to precipitate the development of CAI. The development of this model was a seminal step in facilitating an understanding of why many patients incur repeated ankle joint dysfunction. The use of the term $C A I$ according to the Hertel ${ }^{19}$ model represented the initial attempt to define and provide potential contributions from functional and mechanical insufficiencies, which helped develop a more comprehensive approach to researching and treating patients with this pathology.

Research related to ankle joint instability evolved over the decade following the publication of the Hertel CAI model, ${ }^{19}$ with a primary aim of much of the research devoted to understanding exactly what combinations of functional and mechanical insufficiencies best define CAI. Many recent reviews and multifactorial studies have provided important information outlining that there are multiple potential contributing mechanical, neuromuscular, functional, and/or perceived deficits that may persist long after physiologic tissue healing times have elapsed and interventions have been completed following an acute ankle joint sprain. ${ }^{23-34}$ Consistently, these reviews and multifactorial studies support the proposition that CAI is a multifaceted and complex condition, requiring further indepth interdisciplinary study.

While the volume and quality of this research grew substantially, it became more evident that CAI patients are quite heterogeneous in their presentation of impairments, leading the research toward consideration of a possible conglomeration of subgroups. Recently, Hiller et $\mathrm{al}^{21}$ introduced an evolution of the Hertel CAI model ${ }^{19}$ that suggests there may be as many as 7 different subsets of patients that incur persistent symptoms following an initial ankle joint sprain, which are dependent upon the complex interaction of mechanical insufficiencies, perceived instability, and frequency of recurrent sprains.

\section{RATIONALE}

When one examines the body of work related to repeated and recurrent ankle joint injury and instability, there is a spectrum of patient characteristics that have been used within the ankle instability (including CAI and functional ankle instability) research literature from the last 2 decades. ${ }^{20,21}$ Delahunt et $\mathrm{al}^{20}$ systematically investigated these issues in the research relating to recurrent ankle joint sprain and the resulting inconsistent definitions and use of terms such as CAI, functional ankle instability, etc. They concluded that $C A I$ was the most commonly used term to describe participants who report ongoing symptoms after an initial ankle sprain; and the most commonly reported deficits associated with CAI were frequent/recurrent sprains and episodes of or the reporting of feelings of ankle joint 
1. A history of at least 1 significant ankle sprain

The initial sprain must have occurred at least 12 months prior to study enrollment

Was associated with inflammatory symptoms (pain, swelling, etc)

Created at least 1 interrupted day of desired physical activity

The most recent injury must have occurred more than 3 months prior to study enrollment.

We endorse the definition of an ankle sprain as "An acute traumatic injury to the lateral ligament complex of the ankle joint as a result of excessive inversion of the rear foot or a combined plantar flexion and adduction of the foot. This usually results in some initial deficits of function and disability." 20

2. A history of the previously injured ankle joint "giving way" and/or recurrent sprain and/or "feelings of instability."

We endorse the definition of "giving way" as "The regular occurrence of uncontrolled and unpredictable episodes of excessive inversion of the rear foot (usually experienced during initial contact during walking or running), which do not result in an acute lateral ankle sprain." Specifically, participants should report at least 2 episodes of giving way in the 6 months prior to study enrollment.

We endorse the definition of "recurrent sprain" as two or more sprains to the same ankle..$^{20}$

We endorse the definition of "feeling of ankle joint instability" as "The situation whereby during activities of daily living (ADL) and sporting activities the participant feels that the ankle joint is unstable and is usually associated with the fear of sustaining an acute ligament sprain." 20

Specifically, self-reported ankle instability should be confirmed with a validated ankle instability specific questionnaire using the associated cut-off score. Currently recommended questionnaires:

a. Ankle Instability Instrument (AII) ${ }^{40}$ : answer "yes" to at least 5 yes/no questions (This should include question 1, plus 4 others.)

b. Cumberland Ankle Instability Tool (CAIT) $)^{41}:<24$

c. Identification of Functional Ankle Instability (IdFAI) ${ }^{37}:>11$

3. A general self-reported foot and ankle function questionnaire is recommended to describe the level of disability of the cohort, but should only be an inclusion criterion if the level of self-reported function is important to the research question. Currently endorsed questionnaires:

a. Foot and Ankle Ability Measure (FAAM) ${ }^{42}$ : ADL scale $<90 \%$, Sport scale $<80 \%$

b. Foot and Ankle Outcome Score (FAOS) ${ }^{43}$ : $<75 \%$ in 3 or more categories

"giving way." Subsequently, the authors advocated that research in this area could be improved if consistent terminology and a specific set of patient selection criteria could be established.

\section{STATEMENT OBJECTIVES}

It is the opinion of the International Ankle Consortium that some of the inconsistency in defining the factors and characteristics that best explain recurrent ankle sprains and instability may be attributed to inconsistent inclusion criteria among this literature. The International Ankle Consortium proposes the establishment of an accepted set of selection criteria, which should be used in this area of research, as it will provide consistency to the future data synthesis devoted to improving the understanding of the CAI and enhance external validity of findings for this patient population. The purpose of this position statement is to present and discuss an endorsed set of selection criteria for patients with CAI based on the best available evidence to be used in future research and study designs. Our group wishes to advocate for the pursuit of the strongest and most appropriate evidence that will improve the understanding and management of CAI.

\section{CRITERIA RECOMMENDATIONS}

Standard inclusion and exclusion criteria endorsed by the International Ankle Consortium, as a minimum, for enrolling patients that fall within the heterogeneous condition of CAI in controlled research are listed in Tables 1 and 2. Additionally, the International Ankle Consortium encourages the reporting of critical information found in Table 3 for patients with CAI to provide a comprehensive description of the study participants that have been enrolled in controlled research studies.

\section{DISCUSSION}

The preceding endorsed criteria for selection of CAI patients in research are based on the best available evidence and the International Ankle Consortium recommends adherence in order to produce consistent population characteristics for improved outcomes and external validity in future research of this clinical phenomenon. These recommendations will enhance the validity of research conducted in this clinical population with the end goal of bringing the research evidence to the clinician and patient. Additional rationale for the selection criteria will be provided below.

The International Ankle Consortium acknowledges the work of Delahunt et $\mathrm{al}^{20}$ that has provided the framework for this position statement and recommends consultation of and familiarization with that work by all researchers with an interest in CAI. The aims of the systematic investigation of Delahunt and colleagues ${ }^{20}$ were to (1) identify the definition of ankle instability used by authors publishing research papers pertaining to ankle joint sprain and its subsequent sequelae; (2) identify the terminology utilized by authors to classify participants with chronic ankle instability (eg, CAI, functional ankle instability, mechanical ankle instability, or other); (3) to identify the specific inclusion criteria used by authors publishing research papers pertaining to ankle joint sprain and subsequent sequelae. This was the first published paper to systematically investigate the aforementioned issues, which may lead to inconsistencies in research results relating to ankle joint sprain and its subsequent sequelae. The results of this 
Table 2. Standard Exclusion Criteria Endorsed, as a Minimum, by the International Ankle Consortium for Enrolling Patients that Fall Within the Heterogeneous Condition of Chronic Ankle Instability in Controlled Research

\section{Exclusion Criteria}

1. A history of previous surgeries to the musculoskeletal structures (ie, bones, joint structures, nerves) in either limb of the lower extremity

It is understood and accepted in clinical and research practice that surgery to repair insufficient joint structures is designed to restore structural integrity but creates residual changes in the central and peripheral portions of the nervous system. Even with appropriate rehabilitation and follow-up management, there are concomitant neuromuscular and structural alterations after surgery that would confound the ability to isolate the effects of chronic ankle instability.

2. A history of a fracture in either limb of the lower extremity requiring realignment

Similar to the first exclusion criterion, significant compromise to skeletal tissue will threaten the internal validity of the selection of study populations with isolated chronic ankle instability.

3. Acute injury to musculoskeletal structures of other joints of the lower extremity in the previous 3 months, which impacted joint integrity and function (ie, sprains, fractures) resulting in at least 1 interrupted day of desired physical activity

systematic investigation indicated that $C A I$ was the most commonly used term to describe participants who report ongoing symptoms after an initial ankle sprain. Furthermore, the most commonly used descriptors relating to CAI were frequent/recurrent sprains and episodes of or the reporting of feelings of ankle joint giving way. Based on their findings, Delahunt et $\mathrm{al}^{20}$ recommended that consistent terminology and a specific minimum set of criteria be reported as this would improve research endeavor pertaining to CAI. As such, Delahunt et $\mathrm{al}^{20}$ devised a set of operational definitions relating to ankle joint sprain and its subsequent sequelae, as well as a specific set of criteria that should be reported when undertaking research on participants with CAI. These definitions and criteria set formed the basis of discussion at the International Ankle Symposium, from which the International Ankle Consortium has formed a consensus statement relating to operational definitions pertaining to ankle joint sprain and its subsequent sequelae and a minimum set of criteria to be reported when conducting CAI research.

At the 5th International Ankle Symposium (Lexington, KY, USA, 2012), the International Ankle Consortium Executive Committee discussed the concepts of this position paper based on the existing work and the new information being presented at the meeting. ${ }^{35}$ Consistent with the work by Delahunt et al ${ }^{20}$ new papers presented at the International Ankle Symposium emphasized the strength of reported episodes of giving way and patientreported instability in defining CAI. Snyder et al, ${ }^{36}$ using the Delphi method to gather input from expert clinicians and researchers, reported that "recurrent sense of giving way" was the strongest characteristic in defining CAI. However, there are other characteristics, such as feelings of instability and recovery from a "rolling-over" incident, ${ }^{37}$ that are important in both identifying who has CAI and establishing the severity of the condition that are not obtained through the reporting of giving way alone. A series of papers ${ }^{38,39}$ support the use of condition-specific self-report questionnaires to identify those with the minimal accepted criteria for ankle instability. It is critical to utilize condition-specific questionnaires that are both valid and reliable ${ }^{37,40,41}$ in the collection of this information. This recent work highlights the increasing evidence for the selected criteria we introduced in this position paper.

Additionally, measurement of self-reported instability should be differentiated from measurement of resulting change to physical function or quality of life. Changes to physical function may be a result of any or all of mechanical insufficiencies, self-reported instability, and recurrent sprains. Therefore, if investigators are interested in the deficits present in participants with CAI, such as strength, neuromuscular, or proprioception deficits as examples, measures of self-reported function may not be a necessary inclusion criterion for this type of study. However, if functional impairment is relevant to the proposed project or intervention, then validated anklespecific questionnaires that were designed to evaluate selfreported function should be used to create the necessary inclusion criterion. ${ }^{42,43}$

Our recommended inclusion criteria are based on assessments of injury history, function and disability, but we recognize the lack of definitive selection criteria based on an assessment of joint integrity or laxity. While an initial ankle sprain often threatens the integrity of ligamentous structures and some authors have reported lingering ankle laxity, hypomobility, and hypermobility, these outcomes do not appear to be observed consistently in CAI patients. Previous authors have considered mechanical instability as an explanatory factor for lingering ankle instability, but there has not been a definitive association of ankle laxity with CAI. ${ }^{19-21,23,28,29,33,44,45}$

Hertel's ${ }^{19}$ original model differentiated mechanical instability from functional instability. More recently, Hiller et $\mathrm{al}^{21}$ refining the model of categorizing CAI, suggest as many as 7 subgroups of individuals with CAI that likely provide better homogeneity in describing the pathology. Of the 3 primary separation factors, the authors suggested that mechanical instability provided the weakest contribution. Additionally, hypomobility, rather than joint laxity, contributes more to the subgroup model creation. It appears that mechanical instability may be a factor in some patients that leads to recurrent ankle injury and measures of perceived ankle instability, but these are not necessarily dependent upon the presence of ankle hypermobility. Data from other multifactorial studies that have included measures of mechanical instability in CAI patients suggest that mechanical instability alone is not a consistent identifier of this pathology. ${ }^{28,33}$

A recent advancement in the CAI literature has been the stratification of individuals based on structural and functional impairments associated with ankle instability. Multiple studies by Brown et al ${ }^{44-46}$ compared sensorimotor and biomechanical measures between patients classified as having mechanical ankle instability, functional ankle instability, and copers (no measurable ankle instability or repeated injury). While the presence of mechanical laxity was associated with some proximal-joint sensorimotor alterations and increases in ground reaction forces during landing tasks compared with the other groups, these differences were not observed consistently. It is also interesting to note that the mechanical ankle instability 
Table 3. Information Recommended by the International Ankle Consortium for Patients with Chronic Ankle Instability in Order to Provide a Comprehensive Description of the Study Participants that have been Enrolled in Controlled Research Studies

\begin{tabular}{|c|c|}
\hline Topic & Suggested Content \\
\hline Quality of ankle injury history & $\begin{array}{l}\text { 1. The number of previous ankle sprains } \\
\text { 2. The presence of and frequency of reported episodes of "giving way" } \\
\text { 3. The presence of and frequency of reported episodes of feelings of instability } \\
\text { 4. The scores on the validated self-reported ankle instability instruments utilized to establish inclusion } \\
\text { criteria } \\
\text { 5. Severity of injury (index and most recent incidents), including the number of days of immobilization and/ } \\
\text { or nonweight bearing } \\
\text { 6. If diagnosis was performed by health care professional or self-diagnosed }\end{array}$ \\
\hline Potential confounding factors & $\begin{array}{l}\text { 10. Any included mechanical instability ratings (ie, clinical laxity scales, arthrometry measures, stress } \\
\text { radiography) } \\
\text { 11. A rating of current level of physical activity level using a validated scale (eg, Tegner scale, Godin } \\
\text { Leisure Time Physical Activity), and the minimum number of hours per week of participation in physical } \\
\text { activity } \\
\text { 12. Any concomitant, nonsurgical injuries at the time of ankle sprain } \\
\text { 13. The frequency of use of prophylactic ankle support } \\
\text { 14. The results of any functional or range-of-motion assessments } \\
\text { 15. Presence of pain during functional activities }\end{array}$ \\
\hline
\end{tabular}

groups had more self-reported disability and no differences in the number of episodes of giving way compared with the functional ankle instability groups, suggesting that the mechanical ankle instability groups had similar, if not more, functional instability than the functional ankle instability groups did. The design of these studies to separate mechanical ankle instability and functional ankle instability represents the needed comparisons required to glean the factors that best define CAI. The information would seem to lend support to the strength of the contribution of functional instability measures, rather than mechanical instability, to defining CAI.

\section{FUTURE CONSIDERATIONS}

We have provided recommendations for selection of patients with CAI in order to improve the quality of research on this pathology. The health care burden associated with ankle instability necessitates increased research and clinical outcomes that can be used to reduce the disability and recurrence rates associated with CAI. It is clear from the body of literature that there are many contributing factors to CAI that can create a host of impairments ${ }^{19,20,23,26,28,29,32,33}$; however, this condition is more heterogeneous than many realize. ${ }^{20,21}$ Therefore, researchers need to be cognizant of criteria that are best associated with CAI based on current available evidence. Based on the collective expertise of the International Ankle Consortium, we feel that the specified selection criteria should be incorporated in all future research on CAI.

The selection criteria are based on history of initial injury, history of ongoing bouts of instability, and ratings of patient-perceived function and disability gathered from validated survey instruments. In addition, to study CAI in patients, concomitant issues such as fracture and surgery and other significant lower extremity joint injury should be absent, as well as an appropriate amount of time should have passed since suffering acute, inflammatory symptoms, all for the purpose of eliminating confounding influence on the outcomes that researchers choose to employ.

We have provided our list of additional patient information that we feel should be reported, and we look forward to evaluating and utilizing the evidence that continues to grow from this work to modify our recommendations moving forward. In the future, consistency among these suggested reported measures will only help to strengthen the description and understanding of CAI. In the meantime, researchers should strive to report as many of these data to create clearer descriptions of CAI, which may to more homogeneous subgroups. The rationale for this is to improve the understanding of the consequences of repetitive ankle injury and lingering instability, leading to development of more effective interventions to decrease the acute and chronic ankle injury rates in physically active populations.

\section{STATEMENT AND BACKGROUND OF CREATION OF THE POSITION STATEMENT}

The International Ankle Consortium, formed in 2004, is an international community of researchers and clinicians whose primary scholastic purpose is to promote scholarship and dissemination of research-informed knowledge information related to pathologies of the ankle complex. We are a collegial network that strives to support the ongoing growth of scientific and clinical evidence to elucidate the mechanisms, characteristics, and interventions related to ankle complex and joint pathologies. The International Ankle Symposium is the primary venue by which the International Ankle Consortium disseminates the work of its constituents in an effort to present and discuss the most contemporary theories and research related to ankle joint clinical phenomena and related interventions, with a primary focus on CAI.

Another focus of the International Ankle Consortium is to provide endorsement for standards of clinical research related to ankle joint pathologies. The International Ankle 
Consortium endorses the summary statements from past International Ankle Symposia that have presented the major findings and updates from the content of the meetings. ${ }^{35,47-49}$ Additionally, the International Ankle Consortium establishes position statements, such as this one, to endorse consistent standards for research of and clinical management of ankle joint conditions among the physically active. This position statement will provide background and discusses the existing evidence to support a set of specific selection criteria for patients with chronic/functional ankle instability with the goal to improve the quality of research and outcomes related to this specific ankle condition.

\section{DISCLAIMER}

This document was prepared by the authors and is printed here without correction. The accuracy, nomenclature, form, and style all remain the responsibility of the authors.

\section{REFERENCES}

1. United States Bone and Joint Initiative. The Bone and Joint Decade: The Burden of Musculoskeletal Diseases in the United States. http:// www.boneandjointburden.org/. Accessed November 1, 2013.

2. National Center for Health Statistics, National Ambulatory Medical Care Survey, 2006.

3. Centers for Disease Control and Prevention. National Ambulatory Medical Care Survey (NAMCS), 2006. http://www.cdc.gov/nchs/ ahcd.htm. Accessed November 1, 2013.

4. Centers for Disease Control and Prevention. National Center for Health Statistics. National Health Interview Survey, 2008 Data Release. http://www.cdc.gov/nchs/nhis/nhis_2008_data_release.htm. Accessed November 1, 2013.

5. Waterman B, Owens B, Davey S, Zacchilli M, Belmont P. The epidemiology of ankle sprains in the United States. J Bone Joint Surg Am. 2010;92(13):2279-2284.

6. Cooke M, Lamb S, Marsh J, Dale J. A survey of current consultant practice of treatment of severe ankle sprains in emergency departments in the United Kingdom. Emerg Med J. 2003;20(6):505-507.

7. McKay GD, Goldie PA, Payne WR, Oakes BW. Ankle injuries in basketball: injury rate and risk factors. $\mathrm{Br} J$ Sports Med. 2001;35(2):103-108.

8. Beynnon BD, Murphy DF, Alosa DM. Predictive factors for lateral ankle sprains: a literature review. J Athl Train. 2002;37(4):376-380.

9. Yeung MS, Chan KM, So CH, Yuan WY. An epidemiological survey on ankle sprain. Br J Sports Med. 1994;28(2):112-116.

10. Swenson DM, Yard EE, Fields SK, Comstock RD. Patterns of recurrent injuries among US high school athletes, 2005-2008. Am J Sports Med. 2009;37(8):1586-1593.

11. Hiller CE, Nightingale EJ, Raymond J, et al. Prevalence and impact of chronic musculoskeletal ankle disorders in the community. Arch Phys Med Rehabil. 2012;93(10):1801-1807.

12. Brown T, Johnston R, Saltzman C, Marsh J, Buckwalter J. Posttraumatic osteoarthritis: a first estimate of incidence, prevalence, and burden of disease. J Orthop Trauma. 2006;20(10):739-744.

13. Caine DJ, Golightly YM. Osteoarthritis as an outcome of paediatric sport: an epidemiological perspective. $B r J$ Sports Med. 2011;45(4):298-303.

14. Saltzman CL, Zimmerman B, O'Rourke M, Brown TD, Buckwalter JA, Johnston R. Impact of comorbidities on the measurement of health in patients with ankle osteoarthritis. J Bone Joint Surg Am. 2006;88(11):2366-2372.

15. Knight E, Ofsthun N, Teng M, Lazarus J, Curhan G. The association between mental health, physical function, and hemodialysis mortality. Kidney Int. 2003;63(5):1843-1851.
16. Sneed NV, Paul S, Michel Y, VanBakel A, Hendrix G. Evaluation of 3 quality of life measurement tools in patients with chronic heart failure. Heart Lung. 2001;30(5):332-340.

17. Gage H, Hendricks A, Zhang S, Kazis L. The relative health related quality of life of veterans with Parkinson's disease. J Neurol Neurosurg Psychiatry. 2003;74(2):163-169.

18. Anandacoomarasamy A, Barnsley L. Long term outcomes of inversion ankle injuries. Br J Sports Med. 2005;39(3):e14.

19. Konradsen L, Bech L, Ehrenbjerg M, Nickelsen T. Seven years follow-up after ankle inversion trauma. Scand J Med Sci Sports. 2002;12(3):129-135.

20. Hertel J. Functional anatomy, pathomechanics, and pathophysiology of lateral ankle instability. J Athl Train. 2002;37(4):364-375.

21. Delahunt E, Coughlan GF, Caulfield B, Nightingale EJ, Lin CWC, Hiller CE. Inclusion criteria when investigating insufficiencies in chronic ankle instability. Med Sci Sports Exerc. 2010;42(11):21062121.

22. Hiller C, Kilbreath S, Refshauge K. Chronic ankle instability: evolution of the model. J Athl Train. 2011;46(2):133-141.

23. Freeman MA. Instability of the foot after injuries to the lateral ligament of the ankle. J Bone Joint Surg Br. 1965;47(4):669-677.

24. Hiller CE, Nightingale EJ, Lin CW, Coughlan GF, Caulfield B, Delahunt E. Characteristics of people with recurrent ankle sprains: a systematic review with meta-analysis. $\mathrm{Br} J$ Sports Med. 2011;45(8):660-672.

25. Arnold B, De La Motte S, Linens S, Ross S. Ankle instability is associated with balance impairments: a meta-analysis. Med Sci Sports Exerc. 2009;41(5):1048-1062.

26. Brinkman RE, Evans TA. History of ankle sprain as a risk factor of future lateral ankle sprain in athletes. J Sport Rehabil. 2011;20(3):384-388.

27. Hertel J. Sensorimotor deficits with ankle sprains and chronic ankle instability. Clin Sports Med. 2008;27(3):353-370.

28. Hirai D, Docherty CL, Schrader J. Severity of functional and mechanical ankle instability in an active population. Foot Ankle Int. 2009;30(11):1071-1077.

29. Hubbard T, Kramer L, Denegar C, Hertel J. Contributing factors to chronic ankle instability. Foot Ankle Int. 2007;28(3):343-354.

30. Hubbard-Turner T. Relationship between mechanical ankle joint laxity and subjective function. Foot Ankle Int. 2012;33(10):852-856.

31. McKeon P, Hertel J. Systematic review of postural control and lateral ankle instability, part I: can deficits be detected with instrumented testing. J Athl Train. 2008;43(3):293-304.

32. Munn J, Sullivan S, Schneiders A. Evidence of sensorimotor deficits in functional ankle instability: a systematic review with metaanalysis. J Sci Med Sport. 2010;13(1):2-12.

33. Sefton J, Hicks-Little C, Hubbard T, et al. Sensorimotor function as a predictor of chronic ankle instability. Clin Biomech (Bristol, Avon). 2009;24(5):451-458.

34. Wikstrom EA, Tillman MD, Chmielewski TL, Cauraugh JH, Naugle $\mathrm{KE}$, Borsa P. Discriminating between copers and people with chronic ankle instability. J Athl Train. 2012;47(2):136-142.

35. Witchalls J, Blanch $P$, Waddington $G$, Adams R. Intrinsic functional deficits associated with increased risk of ankle injuries: a systematic review with meta-analysis. Br J Sports Med. 2012;46(7):515-523.

36. McKeon P, Mattacola C. Fifth International Ankle Symposium: October 17-20, 2012, Lexington, Kentucky. J Orthop Sports Phys Ther. 2013;43(3 suppl):A1-A27.

37. Snyder K, Evans T, Neibert P, Weiss W, Haak T. Development of an ankle instability model. Paper presented at: 5th International Ankle Symposium; October 17-20, 2012; Lexington, KY.

38. Simon J, Donahue M, Docherty C. Development of the identification of functional ankle instability (IdFAI). Foot Ankle Int. Sep 2012;33(9):755-763.

39. Donahue M, Simon J, Docherty C. Critical review of self-reported functional ankle instability measures. Foot Ankle Int. 2011;32(12):1140-1146. 
40. Donahue M, Simon J, Docherty C. Critical review of self-reported functional ankle instability measures: a follow-up. Paper presented at: 5th International Ankle Symposium; October 17-20, 2012; Lexington, $\mathrm{KY}$.

41. Docherty C, Gansneder B, Arnold B, Hurwitz S. Development and reliability of the ankle instability instrument. J Athl Train. 2006;41(2):154-158.

42. Hiller C, Refshauge K, Bundy A, Herbert R, Kilbreath S. The Cumberland ankle instability tool: a report of validity and reliability testing. Arch Phys Med Rehabil. 2006;87(9):1235-1241.

43. Carcia C, Martin R, Drouin J. Validity of the Foot and Ankle Ability Measure in athletes with chronic ankle instability. J Athl Train. 2008;43(2):179-183.

44. Roos EM, Brandsson S, Karlsson J. Validation of the foot and ankle outcome score for ankle ligament reconstruction. Foot Ankle Int. 2001;22(10):788-794.

45. Brown C, Bowser B, Simpson KJ. Movement variability during single leg jump landings in individuals with and without chronic ankle instability. Clin Biomech (Bristol, Avon). 2012;27(1):52-63.
46. Brown CN, Padua DA, Marshall SW, Guskiewicz KM. Variability of motion in individuals with mechanical or functional ankle instability during a stop jump maneuver. Clin Biomech (Bristol, Avon). 2009;24(9):762-768.

47. Brown CN, Padua DA, Marshall SW, Guskiewicz KM. Hip kinematics during a stop-jump task in patients with chronic ankle instability. J Athl Train. 2011;46(5):461-467.

48. Hertel J, Kaminski T. Second International Ankle Symposium, October 15-16, Newark, Delaware. J Orthop Sports Phys Ther. 2005;35(5 suppl):A1-A28.

49. Caulfield B. Third International Ankle Symposium, September 1-3, 2006, Dublin, Ireland: introduction, summary statement, list of keynote lecturers and abstracts, keynote lectures, and abstracts. $J$ Orthop Sports Phys Ther. 2006;36(11 suppl):A1-A27.

50. Refshauge K, Hiller C. Fourth International Ankle Symposium, July 16-18, 2009, Sydney, Australia. J Orthop Sports Phys Ther. 2009;39(10 suppl):A1-A24.

Address correspondence to Phillip A. Gribble, PhD, ATC, FNATA, Health and Human Services, The University of Toledo, 2801 W. Bancroft Street, Mailstop \#119, Health and Human Services - 2503, Toledo, OH 43606. Address e-mail to phillip.gribble@utoledo. edu. 